\title{
Abscesos prostáticos y manejo con resección transuretral de próstata
}

\section{Prostatic Abscesses and Prostate Management with Transurethral Resection}

\author{
Walter Bacaret Cárdenas ${ }^{1}$ Andrés Felipe Puentes ${ }^{1}$ Sebastián Rodríguez Piraquive ${ }^{1}$ Héctor Corredor ${ }^{1}$ \\ Cesar Andrés Capera ${ }^{1}$ Wilfredo Donoso ${ }^{1}$ \\ 1 Departmento de Urología, Universidad Nacional de Colombia, \\ Bogotá, Colombia \\ Urol Colomb 2019;28:275-278. \\ Address for correspondence Walter Bacaret, Residente de Urología, \\ Departamento de Urología, Universidad Nacional de Colombia, Kra \\ 59bbis \#128b 07 Bloque 4 Apto 101, Bogotá, Colombia \\ (e-mail: bacaret666@hotmail.com).
}

\section{Resumen}

\section{Palabras Clave}

- absceso prostático

- tratamiento

- resección transuretral de próstata
Objetivos Caracterizar una serie de pacientes con abscesos prostáticos tratados con resección transuretral de próstata, analizar los factores de riesgo, y los resultados del tratamiento.

Métodos Se realizó un estudio retrospectivo de 2015 a 2016 en 2 hospitales de III y IV nivel en la ciudad de Bogotá en pacientes con diagnóstico tomográfico de absceso prostático, tratados con resección transuretral de próstata.

Resultados Se documentaron 6 casos de hombres diagnosticados con absceso prostático con edad promedio de 57 años (40-78 años) que fueron llevados a RTUP para el drenaje del absceso. El promedio de hospitalización fue de 11.4 días (3-34 días); el aislamiento microbiológico positivo se alcanzó en el 83\% de los pacientes. El 50\% de los pacientes usaban sonda uretral a permanencia. La curación fue completa.

Conclusiones La RTU de próstata en el drenaje de abscesos prostáticos, es un tratamiento seguro y eficaz en logrando resolución completa de la enfermedad.

Objectives To characterize patients with prostate abscesses treated with transurethral resection of the prostate, analyze risk factors, and treatment outcomes. Methods A retrospective study was conducted from 2015 to 2016 in 2 hospitals of III and IV level in the city of Bogotá in patients with tomographic diagnosis of prostate abscess, treated with transurethral resection of the prostate.

Results Six cases of men diagnosed with prostate abscess with an average age of 57 years (40-78 years) who were taken to TURP for drainage of the abscess were documented. The average hospitalization was 11.4 days (3-34 days); Positive microbiological isolation was achieved in $83 \%$ of patients. $50 \%$ of patients used a urethral catheter permanently. The healing was complete.

Conclusions The Transurethral resection of the prostate is a safe and effective intervention in achieving complete resolution and drainage of prostatic abscesses. received

February 2, 2018

accepted

March 25, 2018
DOI https://doi.org/

$10.1055 / \mathrm{s}-0038-1648239$.

ISSN 0120-789X.

e ISSN 2027-0119.
Copyright $($ C 2019, Sociedad Colombiana License terms de Urología. Publicado por Thieme Revinter Publicações Ltda., Rio de Janeiro, Brazil. Todos los derechos reservados.

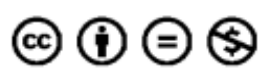




\section{Introducción}

El absceso prostático es una entidad poco prevalente que se presenta entre la quinta y sexta década de la vida como consecuencia de la infección prostática. La incidencia es $0,5 \%$ de todas las enfermedades prostaticas. ${ }^{1}$ La mayoría ocurre en pacientes con factores predisponentes como el uso de sonda uretral a permanencia, retención crónica de orina, prostatitis crónica o inmunodeficiencias. ${ }^{2}$ Se ha reportado la presencia de absceso prostático secundario a biopsia transrectal de próstata la cual tiene incidencia muy baja. Es una patología seria en la que el retraso en su diagnóstico puede producir sepsis secundaria, ruptura del absceso y drenaje a la fosa isquiorectal, choque séptico y muerte. ${ }^{3}$

Los signos y síntomas del absceso prostático incluyen: retención urinaria aguda, fiebre, disuria, frecuencia, dolor perineal, hematuria, secreción uretral o dolor lumbar, y los hallazgos clásicos al examen físico rectal son: sensibilidad y masa renitente. La prueba de oro para el diagnóstico de esa enfermedad es la ultrasonografía transrectal de próstata, son útiles para evaluar extensión prostática y otros posibles focos la tomografía y la resonancia magnética. Los más comunes agentes etiológicos son la Escherichia coli y Staphylococcus aureus, ${ }^{2,3}$ se han reportado casos de infecciones fúngicas por cándida sp y en el pasado, durante la era preantibiótica, se tenía como uno de los agentes etiológicos principales Neisseria gonorrhoeae.

Se ha identificado una variante poco frecuente de la enfermedad conocida como absceso prostático enfisematoso, descrita por primera vez por Mariani y colaboradores en 1983, la cual se define y diagnostica con estudios imagenológicos. Las infecciones urinarias formadoras de gas son altamente mortales, con tasas de letalidad entre el $70 \%$ y el $90 \%$, que para el caso específico del absceso prostático enfisematoso llega hasta un $25 \%{ }^{4}$

El tratamiento consiste en administrar antibióticos en altas dosis y el drenaje del absceso, el cual puede ser punción perineal, resección transuretral o aspiración con aguja guiada por ecografía transrectal. Algunas series de casos y estudios comparativos reportan su experiencia con una u otra técnica mostrando sus riesgos y beneficios, siendo en general la mejor opción, la resección transuretral, mostrando una disminución en las tasas de recurrencia, días de estancia hospitalaria y mortalidad. 5,6

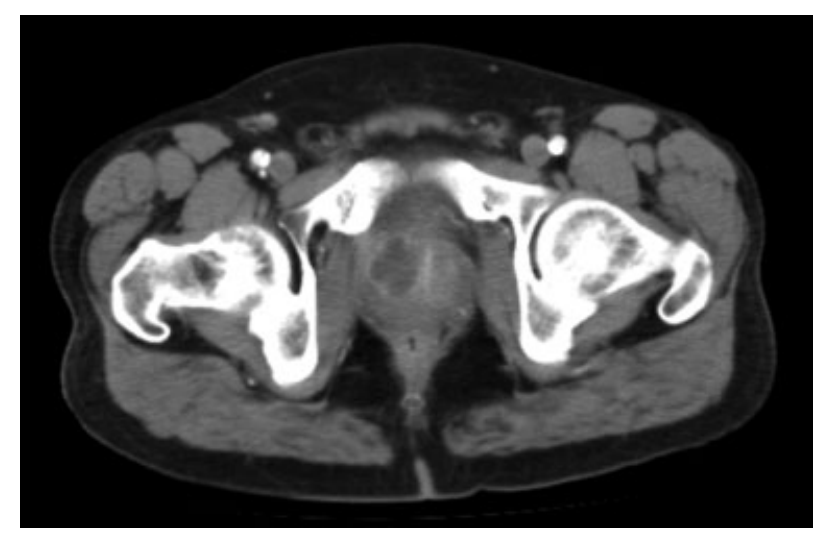

Fig. 1 Absceso prostático visualizado en corte axial.

\section{Materiales y Métodos}

Se realizó un estudio retrospectivo durante los años 2015 a 2016 , recolectando la experiencia en el manejo de abscesos prostáticos mediante la técnica de resección transuretral de próstata (RTU) por el servicio de urología de 2 hospitales de III nivel en la ciudad de Bogotá, los procedimientos fueron realizados mediante la misma técnica de resección transuretral clásica de Mauermeyer, con un resectoscópio calibre 26 Fr, irrigando con Glicina 1,5\%, corriente monopolar entre 60 y $80 \mathrm{~W}$ tanto para corte como para coagulación. Posteriormente, todos los pacientes se mantuvieron con irrigación de 12 a 36 horas y sonda uretral de calibre 22 Fr de 3 vías por 1 semana. El diagnostico de absceso prostático se confirmó en todos los casos mediante tomografía de abdomen contrastado (-Figs. 1 y 2 ).

\section{Resultados}

Se evaluaron los casos de 6 hombres entre los años 2015 y 2017 en dos hospitales de III y IV nivel en la ciudad de Bogotá, quienes se interconsultaron al servicio de urología con el diagnóstico de absceso prostático visualizado en TAC de abdomen contrastado o ecografía de vías urinarias, con un promedio de edad de 57 años (40-78 años), a quienes se les realizó drenaje del absceso mediante la técnica de resección transuretral de próstata, con el objeto de llevar a cabo un destechamiento y drenaje de la colección como se describe en materiales y métodos.

Se encontró un promedio de días de estancia hospitalaria de 28,5 días (8-73 días) y un promedio de hospitalización de 11,4 días (3-34). Posterior a la cirugía, se aisló germen en hemocultivos y urocultivo en el $83 \%$ ( $5 \%$ pacientes) de los casos, con el objetivo de dirigir la terapia antibiótica, la cual fue en su mayor parte, carbapenemicos dada la naturaleza de la resistencia de las bacterias encontradas. No se encontró en ninguno de los casos, infecciones por hongos ni otro tipo de microorganismos. Cabe resaltar, que el diagnostico de absceso se realizó en su mayoría por descarte en pacientes con foco séptico persistente no claro, en quienes se realizó TAC de abdomen y pelvis contrastado en busca de focos ocultos.

El $50 \%$ de los pacientes eran usuarios de sonda a permanencia por patología obstructiva prostática ya sea maligna o benigna, en todos los casos, se observa que los pacientes cursan con patologías de base que comprometen su estado nutricional e inmunológico lo que los hace susceptibles de infecciones en sitios no comunes. Se logró una tasa de curación del $100 \%$ con esa técnica de drenaje, sin ninguna complicación Intra o post operatoria.

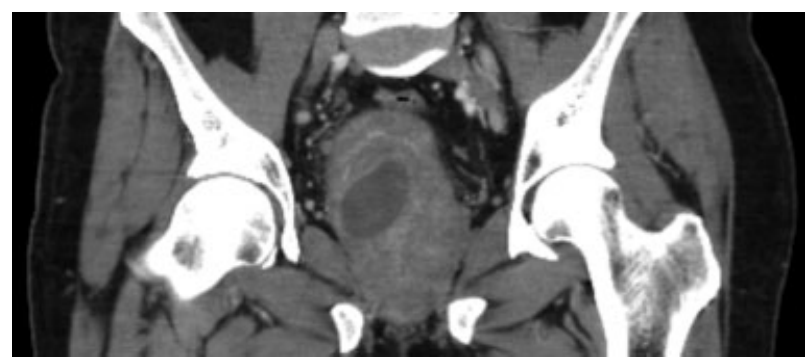

Fig. 2 TAC de abdomen corte coronal. 
Tabla 1 Características generales

\begin{tabular}{|c|c|c|c|c|c|c|}
\hline Paciente & Edad & Comorbilidades & $\begin{array}{l}\text { Días de } \\
\text { hospitalización } \\
\text { total }\end{array}$ & $\begin{array}{l}\text { Días de } \\
\text { hospitalización } \\
\text { posterior a } \\
\text { la cirugía }\end{array}$ & $\begin{array}{l}\text { Organismo } \\
\text { aislado }\end{array}$ & $\begin{array}{l}\text { Manejo } \\
\text { Antibiótico }\end{array}$ \\
\hline 1 & 61 & $\begin{array}{l}\text { Sonda uretral permanente, } \\
\text { Gangrena de Fournier }\end{array}$ & 10 & 6 & E. Cli BEE & $\begin{array}{l}\text { Piperacilina / } \\
\text { tazobactam- } \\
\text { Ertapenem }\end{array}$ \\
\hline 2 & 40 & $\begin{array}{l}\text { Diabetes Mellitus tipo 2, } \\
\text { Esplenctomía } \\
\text { Inmunodeficiencia } \\
\text { en estudio }\end{array}$ & 8 & 3 & S. Hominis & Ertapenem \\
\hline 3 & 78 & $\begin{array}{l}\text { Sonda uretral permanente, } \\
\text { cáncer de próstata }\end{array}$ & 17 & 11 & $\begin{array}{l}\text { E. coli- k. } \\
\text { Oxytoca KPC }\end{array}$ & $\begin{array}{l}\text { Ampicilina / } \\
\text { sulbactam-cefepime- } \\
\text { Meropenem }+ \\
\text { polomixina }\end{array}$ \\
\hline 4 & 56 & Cor-pulmonale, EPOC & 73 & 34 & S. aureus & daptomicina \\
\hline 5 & 46 & Enfermedad Renal Crónica & 45 & 11 & No aislado & $\begin{array}{l}\text { Ertapenem }+ \\
\text { Vancomicina }\end{array}$ \\
\hline 6 & 65 & $\begin{array}{l}\text { Hipertensión arterial, } \\
\text { crecimiento } \\
\text { prostático Benigno, } \\
\text { Sonda a permanencia }\end{array}$ & 18 & 3 & k. Oxytoca KPC & $\begin{array}{l}\text { Meropenem + } \\
\text { cloistina }\end{array}$ \\
\hline
\end{tabular}

\section{Discusión}

En el estudio de Jang y col., se evalúan 52 pacientes en un periodo de 10 años, diagnosticados con tomografía de abdomen con un promedio de edad de 61 años. Once pacientes fueron manejados de manera conservadora, 23 con RTU de próstata y 18 con drenaje con aguja guiado por ecografía trans-rectal. Cuatro pacientes de los manejados con drenaje con aguja, presentaron recurrencia y 2 pacientes manejados de manera conservadora murieron. En nuestro trabajo, se realizó una única técnica de drenaje de absceso, logrando una tasa del $100 \%$ de éxito, sin recurrencias ni muertes. Tampoco se observaron complicaciones secundarias al manejo realizado.

En general, fue un procedimiento bien tolerado que en algunos casos se realizó con el paciente inestable hemodinámicamente, que fue interconsultado durante su estancia en la unidad de cuidado intensivo, y luego de numerosos estudios y terapias fallidas, se llevó a quirófano con todas las consideraciones necesarias, tanto por urología, como por anestesiología y cuidado intensivo, logrando drenar de manera adecuada, el absceso y consiguiendo una mejoría vertiginosa en su estado clínico que permitió rápidamente, retirar los soportes vasoactivos y ventilatorios y prontamente el egreso de la unidad de cuidado intensivo y posterior egreso hospitalario.

Si observamos la - Tabla 1, encontramos que tan solo un paciente tuvo un periodo prolongado de estancia hospitalaria posterior a la cirugía. Es bueno aclarar, que dicho paciente cursaba con abscesos en múltiples órganos los cuales debieron ser tratados hasta lograr el control de todos los focos, por lo que se mantuvo hospitalizado a pesar de la resolución de su patología prostática posterior al adecuado drenaje.
Todos los pacientes recibieron manejos antibióticos indicados por el servicio de infectología con recomendaciones de acuerdo al antibiograma y siendo esquemas que iban desde los 10 hasta los 21 días en la mayoría de los casos. En 3 de los 6 casos, fueron completados los esquemas mediante hospitalización domiciliaria y valorados en consulta postoperatoria al término del manejo antimicrobiano, confirmado con la negatividad del urocultivo y la resolución de la sintomatología. Solo se le realizó tomografía de abdomen y pelvis de control a 2 de los pacientes, confirmando la resolución de la patología.

\section{Conclusiones}

En nuestra experiencia, la RTU de próstata como técnica de drenaje, es un método seguro y eficaz en el manejo de los abscesos prostáticos, logrando un abordaje endoscópico por la vía natural, sin tener contacto con la flora rectal, ni complicaciones derivadas del abordaje endoscópico uretral, todo eso respaldado por la evidencia actual que respalda nuestra iniciativa.

Conflicto de intereses

Los autores declaran no tener ningún conflicto de intereses.

\section{Referencias}

1 Arrabal-Polo MA, Jimenez-Pacheco A, Arrabal-Martin M. Percutaneous drainage of prostatic abscess: case report and literature review. Urol Int 2012;88(01):118-120

2 Dubos M, Barraud O, Fedou AL, et al. Prostatic abscesses and severe sepsis due to methicillin-susceptible Staphylococcus aureus producing Panton-Valentine leukocidin. BMC Infect Dis 2014; $14: 466$ 


\section{Abscesos prostáticos y manejo con resección transuretral de próstata Bacaret y col}

3 Dell'atti L. Prostatic abscess after transrectal ultrasound-guided prostate biopsy. Case report G Chir Vol. 34 - n. 9/10 - pp. 260262,2013

4 Hsu LN, Chiang PH, Kang CH. Emphysematous prostatic abscess: rare case and systematic review. J Formos Med Assoc 2015;114 (03):292-293
5 Chou YH, Tiu CM, Liu JY, et al. Prostatic abscess: transrectal color Doppler ultrasonic diagnosis and minimally invasive therapeutic management. Ultrasound Med Biol 2004;30(06):719-724

6 Jang K, Lee DH, Lee SH, Chung BH. Treatment of prostatic abscess: case collection and comparison of treatment methods. Korean J Urol 2012;53(12):860-864 